\title{
Rhinoplasty as an Adjunct to Facial Rejuvenation
}

\author{
Grant S. Hamilton, III, MD ${ }^{1}$ \\ ${ }^{1}$ Department of Otorhinolaryngology, Mayo Clinic, Rochester, \\ Minnesota \\ Facial Plast Surg 2021;37:211-217.
}

\begin{abstract}
Address for correspondence Grant Hamilton, MD, Department of Otorhinolaryngology, Mayo Clinic, 200 First St. SW, Rochester, MN 55905

(e-mail: Hamilton.Grant@mayo.edu; granthamilton@mac.com).
\end{abstract}

\begin{abstract}
Rhinoplasty typically is not considered a part of the repertoire facial rejuvenation surgery but the same involutional changes that affect the other structures of the face

Keywords

- rhinoplasty

- aging

- nose

- rejuvenation also impact the nose and midface. Comparatively little has been written about nasal rejuvenation. Rhinoplasty may be an under-recognized but a useful adjunct to the rejuvenation of the aging face. The aging nose presents unique pathology due to the involutional changes that affect the cartilage and supporting structures. A comprehensive facial rejuvenation plan should address these progressive effects.
\end{abstract}

Rhinoplasty typically is not considered a part of the repertoire facial rejuvenation surgery but the same involutional changes that affect the other structures of the face also impact the nose and midface. Comparatively little has been written about nasal rejuvenation. A PubMed search at the time of this writing identified 4,038 manuscripts when searching for "facelift" and 3,928 for "blepharoplasty." In contrast, a search for "aging nose" returned 1,277 results and many of those were related to olfaction, respiration, or the paranasal sinuses. Rhinoplasty may be an under-recognized but useful adjunct to rejuvenation of the aging face.

\section{Anatomy}

The structure of the nose is comprised of bone and cartilage with lesser contributions from skin, fibrofatty tissue, and soft tissue connections between the cartilages. The frontal and alveolar processes of the maxilla, nasal bones, and the perpendicular plate of the ethmoid bone provide bony support of the nose. These bones bound the piriform aperture. The anterior nasal spine is in the midline of the alveolar process of the maxilla.

There are five primary cartilages in the nose that are all joined. The paired upper lateral cartilages are joined to the quadrangular cartilage of the septum in the midline and to the lower lateral cartilages at the scroll. A variable number of sesamoid and accessory cartilages extend from the lateral edges of the upper and lower lateral cartilages out to the piriform aperture.

Between the cartilages there are suspensory soft tissue attachments. Soft tissue over the scroll further reinforces the junction of the upper and lower lateral cartilages. The interdomal ligament spans the domes of the lower lateral cartilages whereas the intercrural ligament connects the medial and cephalic edges of the lower lateral cartilages along their length. The piriform ligament joins the lateral crus and accessory cartilages to the frontal process of the maxilla. In the area of the scroll there is a longitudinal scroll ligament that connects the upper and lower lateral cartilages and a vertical scroll ligament that extends outward to the soft tissue envelope of the nose. The piriform aperture ligament affixes the soft tissue envelope to the piriform aperture ${ }^{1}$ ( $\mathbf{\text { Fig. }} \mathbf{1}$ ). Of note, historical manuscripts described a ligamentous attachment from the medial crural feet to the caudal septum. ${ }^{2,3}$ However, Daniel's more recent dissections dispute its existence. ${ }^{1}$

\section{Pathophysiology}

As the facial skeleton ages bone is remodeled with continued growth in some areas and resorption in others. ${ }^{4-8}$ In the midface bone is primarily resorbed with aging. ${ }^{6}$ This loss of support and volume underlies many of the involutional changes that are typical of the aging nose. Although edentulous patients have accelerated rates of maxillary alveolar resorption, this process also occurs in older patients with full dentition-though to a lesser extent. ${ }^{9}$ Shaw and Kahn quantified the changes in the projection of the paranasal area using CTscans and found that the maxilla and glabella lost projection with advancing age and the piriform aperture increased in size $^{6}$ (- Fig. 2). The enlargement of the piriform aperture is consistent with the loss of bony volume in the maxilla. published online March 5, 2021
Issue Theme Facial Rejuvenation: A Multidimensional and Multimodal Perspective; Guest Editor: Fred G. Fedok, MD, FACS (c) 2021. Thieme. All rights reserved. Thieme Medical Publishers, Inc., 333 Seventh Avenue, 18th Floor, New York, NY 10001, USA
DOI https://doi.org/ 10.1055/s-0041-1725108. ISSN 0736-6825. 


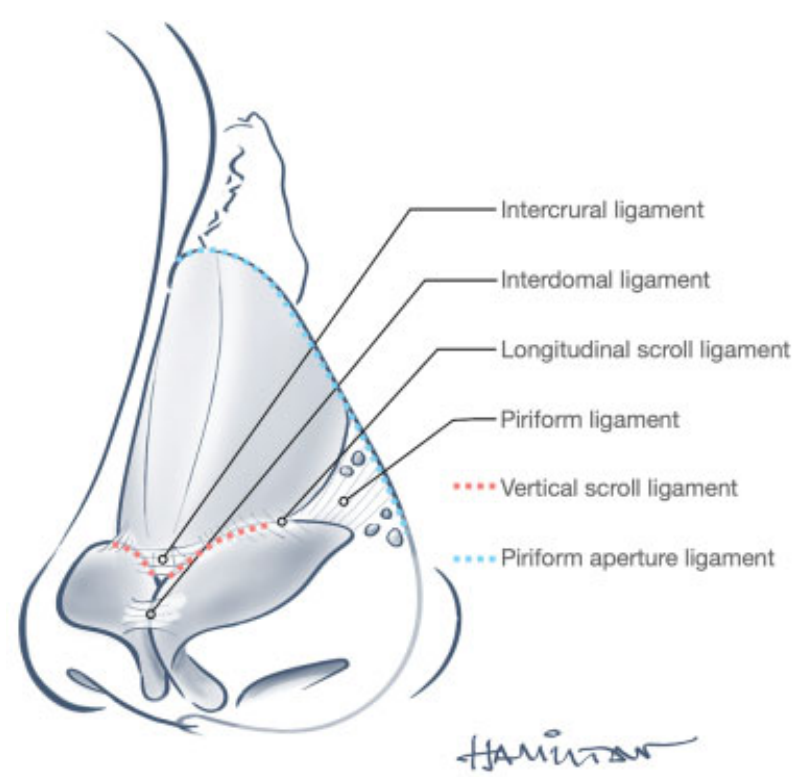

Fig. 1 Ligaments of the nose.

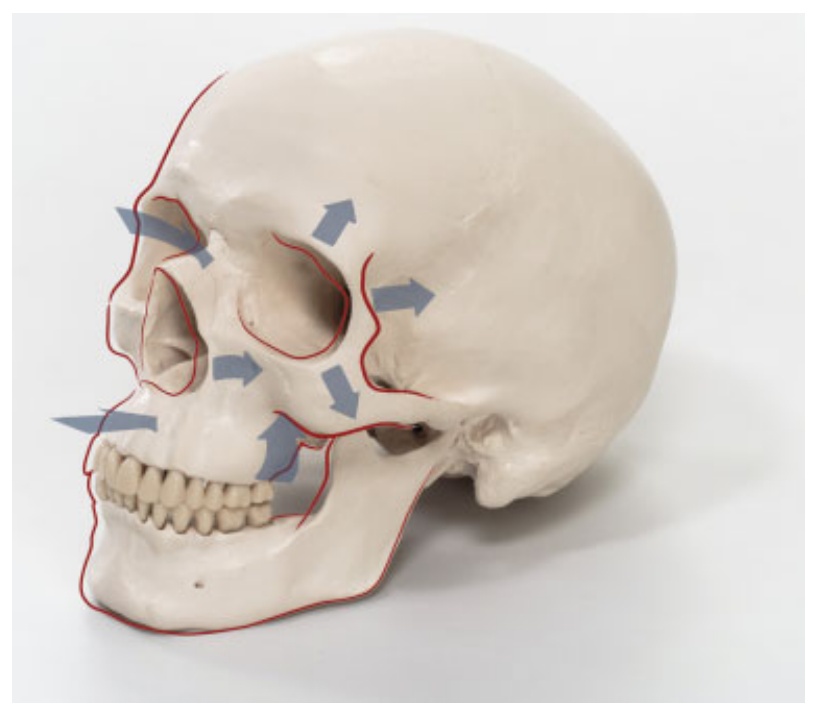

Fig. 2 The facial skeleton partially resorbs with age. The piriform aperture and orbits enlarge while the maxilla and nasion lose anterior projection. In this figure, the red lines indicate the location of the facial bones in youth. The arrows show the direction of resorption.

Recession of the frontal processes of the maxilla seems to be primarily responsible for this increase in area. ${ }^{10}$ Atrophy of the frontal processes underlies the increased prominence of the nasolabial folds seen with aging. ${ }^{11}$ Significant loss of projection also occurs along the base of the piriform apertureincluding the anterior nasal spine. ${ }^{6,10}$ Bone loss in these areas contributes to the decreased support of the nasal base and columella resulting in tip ptosis, nasal lengthening, and a relative retraction of the columella. ${ }^{12,13}$

In addition to the macroscopic structural changes, the nasal cartilages and ligamentous supports undergo microscopic senescent transformation. Helal et al studied the histologic changes in cartilage and soft tissue attachments from patients undergoing rhinoplasty. They found that patients older than 40 had a decrease in the number of chondrocytes and fibrinoid degeneration of the cartilage matrix. Their soft tissue attachments had fewer blood vessels and collagen bundles. ${ }^{14}$ The degree of these histologic changes correlated with anthropometric measurements consistent with the aging changes described above. Other authors have confirmed that weakening of the lower lateral cartilages and decreased integrity of the vertical scroll ligament lead to counter-rotation of the nasal tip, decreased projection, and an increasingly acute nasolabial angle. ${ }^{15}$

The soft tissue envelope is also subject to degenerative changes with aging. The skin loses elasticity, and the epidermis thins. Epidermal cell turnover is slowed which may contribute to delayed wound healing. Collagen bundles decrease and subdermal fat atrophies. ${ }^{16,17}$ Acne rosacea may lead to rhinophyma with hypertrophy of sebaceous glands and thickening of the dermis. ${ }^{18}$ Severe cases can lead to external nasal valve narrowing. ${ }^{19}$

\section{Preoperative Planning}

Older rhinoplasty patients should be evaluated in much the same way as younger ones. The consultation should emphasize the goals of surgery and include some discussion of nasal breathing. Older patients may have different motivations for surgery and may be more likely to be motivated by external factors. ${ }^{20}$ I have had a few patients who sought rhinoplasty after divorce or the death of a spouse who disapproved of the idea of rhinoplastic surgery.

The loss of support in the lower third of the nose often results in aberrant airflow and an increase in the work of breathing. ${ }^{12}$ Elevation of the nasal tip can redirect the inspired air and decrease nasal resistance. ${ }^{21}$ Older patients are more likely to have medical comorbidities and may be anticoagulated. A thorough medical and medication history will identify potential pitfalls that could predispose them to complications.

The physical exam should focus on both the external and internal nose. Aesthetic nasal analysis is performed in the standard way with an overall assessment of nasal and facial proportions. Examine the skin thickness and elasticity. Involutional changes in the vertical scroll ligament may expose more of the middle third of the nose resulting in a disrupted brow-tip aesthetic line.

From the front, there may be some narrowing of the radix due to compensatory elevation of the brow due to ptosis. If this is a concern, a browlift or neurotoxin to elevate the brows is more appropriate solution than surgery in the radix. Conversely, there may be hyperdynamic contraction of the procerus and corrugator muscles resulting in a horizontal crease at the root of the dorsum. Treatment with neurotoxin may be sufficient but a radix graft is indicated if there is an underlying structural deficit. Increased senile bulbosity of the tip due to either cartilage or skin changes (or both) may be present. Deprojection of the tip can widen the interalar distance and cause flaring of the nostrils. Support of the nasal base and reprojection of the tip may be all that is needed to restore the nostril shape. This should be done before performing an alar base reduction. 


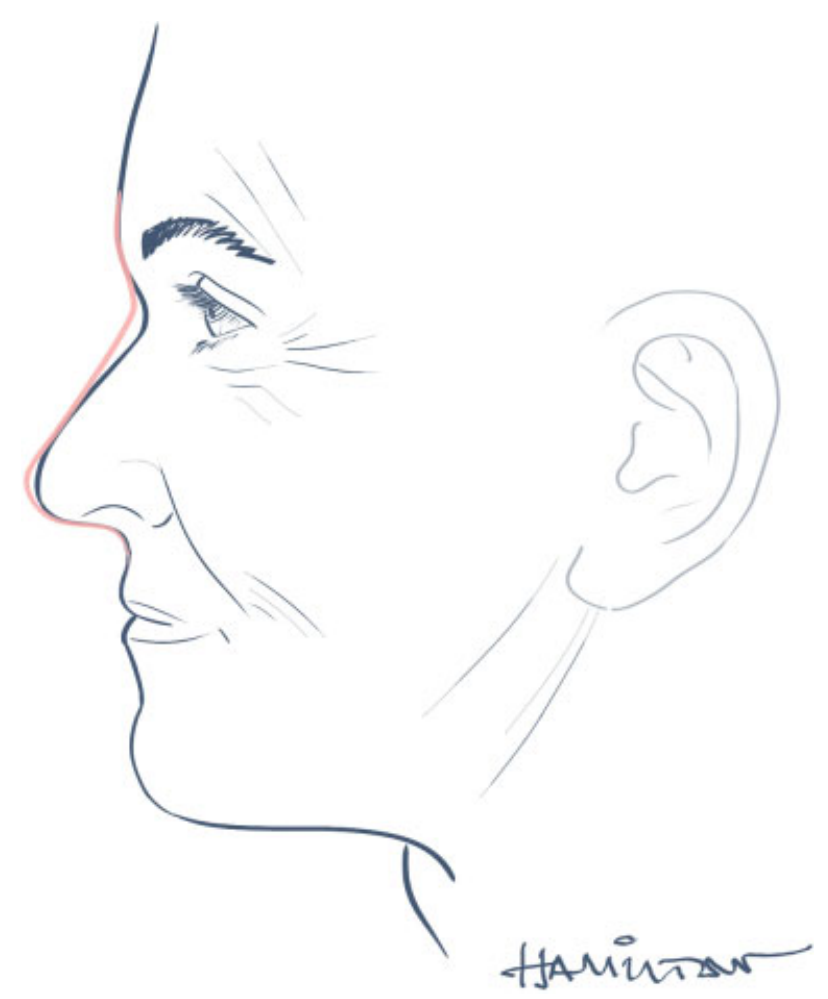

Fig.3 Involutional changes to the cartilage and supporting ligaments may create an appearance of a dorsal convexity. This may be a pseudo dorsal hump. The best solution may be to augment the radix and rotate and project the tip.

On the profile view, look for deepening of the radix and deprojection and counter-rotation of the tip. These changes often create the appearance of a pseudo hump ( - Fig. 3). Though it may be tempting to reduce a dorsal prominence in the aged nose, many times the better solution is to augment the radix and rotate and project the tip. Reducing the pseudo hump alone will result in a straighter profile but the nose will appear to start and finish too low and will look disproportionately long. Rotate and support the tip with a finger to see the effect that this has on the profile and the nasal breathing. Bone resorption along the premaxilla causes retrusion and lengthening of the upper lip and a deepening of the nasolabial angle as the columella settles posteriorly. Usually, reprojection of the base of the nose is sufficient to correct this disproportion but, in some cases, a piriform or premaxillary graft is helpful to compensate for the bony deficiency. ${ }^{10,22}$ This may be especially helpful in edentulous patients. Note that dentures are usually removed for intubation. The upper plate should be replaced after the airway is secured. Without the denture it is impossible to assess the nasolabial angle intraoperatively.

Despite the cellular changes that occur with aging, if patients have not had a septoplasty or rhinoplasty before, the existing septum is a good source of donor cartilage. In the cartilage-depleted patient auricular, costal, and irradiated homograft cartilage can be used. Costal cartilage becomes more brittle and calcified as patients get older (-Fig. 4). Nevertheless, I have successfully used costal cartilage in patients who were in their late seventies. The peripheral layer of costal cartilage resists calcification longer and main-

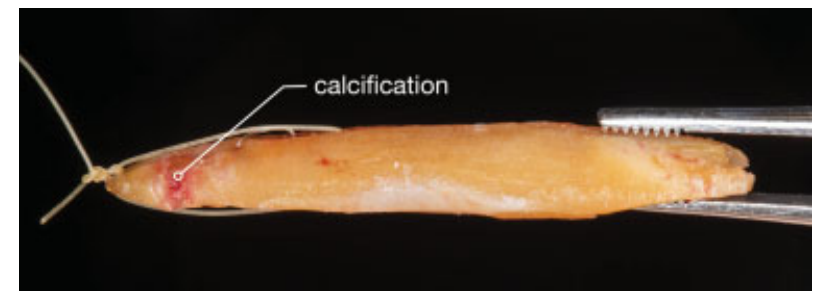

Fig. 4 Costal cartilage becomes yellow, brittle, and calcified with advancing age.

tains its elasticity. This part of the rib is excellent for lateral crural strut grafts and extension of the caudal septum.

\section{Treatment}

\section{Minimally Invasive Options}

Recently, sutures have been proposed as a technique for support and elevation of the nasal tip and valves. ${ }^{23-27}$ These sutures may be permanent or resorbable and are anchored to the radix extending into the superficial musculoaponeurotic system or tip cartilages. The absorbable sutures are typically made of polydioxanone that have small cones that grip the soft tissue to provide lift. The primary advantage to suture lifting of the nose is that the simplified percutaneous access shortens procedure time and speeds recovery when compared to rhinoplasty. Suture lifting with absorbable sutures is not expected to have permanent results but there is some evidence that the results can last a year or longer. ${ }^{26}$

Fillers can also be used either alone or in conjunction with sutures. ${ }^{26}$ To elevate the tip, filler is placed in the nasolabial angle and columella. ${ }^{28,29}$ Filler around the anterior nasal spine creates a platform for elevation of the tip while injections into the columella act like a columellar strut. ${ }^{28}$ Placement of filler in the tip lobule can increase tip projection and definition. Though filler injection into the nose is generally safe when performed properly, complications are possible. These include minor problems like asymmetry or bruising, more concerning complications such as granuloma formation and catastrophic misadventures involving intra-arterial emboli and vascular compromise. $^{28}$

\section{Surgical Options}

\section{Supporting the Nasal Base}

Supporting the nasal base is literally and figuratively the foundation of rhinoplasty in the older patient. The most noticeable age-related change to the nose is the deprojection and counter-rotation of the nasal tip. There are several methods of supporting the nasal base that can be used alone or in combination.

A columellar strut is a strip of cartilage that is sewn between the medial crura to increase the rigidity of the media crural complex. Typically, it is not fixed to the anterior nasal spine for concern about postoperative clicking as the graft slides back and forth over the spine with manipulation of the nose. Daniel proposes suturing the intermediate crura to a columellar strut and affixing the medial crura to the 
caudal septum in a tongue-in-groove manner to support the tip. ${ }^{1}$ When patients have a very heavy, ptotic tip a columellar strut may not be strong enough to rotate and project the nose.

In some cases, it may be possible to overlap the medial crura onto the caudal septum and affix them with a suture. This tongue-in-groove technique is most likely to be an option in patients who have an excess of caudal septal length or who are being deprojected. In those situations, overlapping the medial crura onto the caudal septum will create a favorable relationship. In most patients, however, attaching the medial crura to the caudal septum will result in overrotation and shortening of the nose. ${ }^{1}$

This shortcoming can be avoided by using a caudal extension graft to add enough septum such that it can be placed between the medial crura without creating a foreshortened nose. Caudal extension grafts can be placed inline with the native septum or sewn alongside it. If the nose is already straight, placing it inline will avoid deviating the tip to one side. There are several ways to place a caudal extension graft inline with the septum. One way is to place spreader grafts that extend caudally past the edge of the caudal septum and affix the extension graft between them. Similarly, the extension graft can be attached to the septum with two small splinting grafts ( - Fig. 5). Multiple figures of eight sutures between the graft and the caudal septum is a good option when there is a

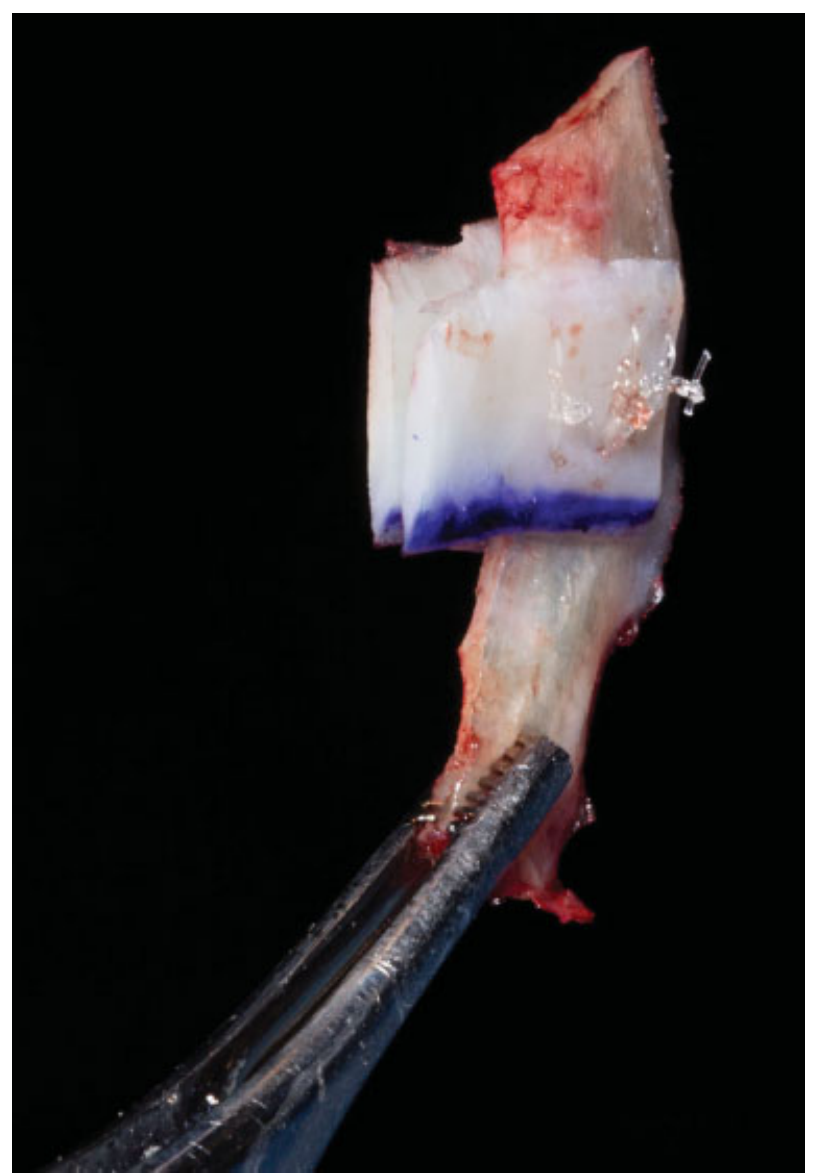

Fig. 5 Two small splinting grafts can be used to anchor an inline caudal extension graft to the caudal septum. paucity of donor cartilage and spreader or splinting grafts are not an option. ${ }^{30} \mathrm{~A}$ caudal extension graft placed side-to-side on the septum is advantageous if the caudal septum needs flattening or reinforcement. In that position it will also act as a septal batten graft.

A caudal extension graft is typically triangular with the apex placed toward the anterior nasal spine. In cases where there is a severely retracted columella the graft may be a trapezoid so that the extended septum can push the columella caudally. Once it is affixed to the caudal septum, it is necessary to divide some of the soft tissue attachments between the medial crura to create a pocket for the graft. When placing the medial crura on the graft it is helpful to use a short needle to temporarily fix them together. The skin can be redraped and the tip position assessed. It is also important to examine the shape of the infratip lobule. It may be necessary to place a temporary suture to close the incision in order to adequately assess the contour of the base of the nose. If the infratip lobule is too flat but the tip is in good position a small onlay graft can be placed over the intermediate crura after permanent fixation. Once the tip position is set, place a suture between the medial edges of the medial crura and the extension graft. Attaching the medial crura to a caudal septal extension graft provides a stable foundation for the nasal tip and precise control of tip position.

\section{Management of the Lateral Crura}

Some of the most significant senescent changes to the nose involve the lateral crura and their attachments. Supporting the base of the nose and repositioning the lower lateral cartilages may be inadequate to appropriately rotate the tip. Shortening the lateral crura is a powerful way to create cephalic rotation of the nose. Note that when used alone, overlapping the lateral crura will deproject the tip a little. ${ }^{31}$ When used in conjunction with a caudal extension graft the tip can be rotated and projected simultaneously.

To overlap the lateral crura, mark symmetric points on the lateral crura. Foda and Kridel recommend planning the incision somewhat laterally but I typically divide the lateral crura $5 \mathrm{~mm}$ lateral to the dome. ${ }^{31}$ Only in the thinnestskinned patients would I move it more laterally. Next, inject a small amount of local anesthetic between the lateral crus and the vestibular skin to hydrodissect them from one another. Use a scalpel to carefully incise the cartilage without cutting the underlying skin. Dissect the skin free from the undersurface of the cartilage for $3 \mathrm{~mm}$ in each direction using fine scissors. Overlap them the necessary amount and suture them with an absorbable monofilament suture. Overlapping the lateral crura has the added advantage that it increases the strength of the lateral crus. ${ }^{32}$

Another option to increase rotation is a lateral crural steal. This has the added benefit that the projection is also increased. ${ }^{31}$ The lateral crural steal is performed similarly to the lateral crural overlay. But instead of dividing the lateral crura they are sutured-moving the dome laterally. Identify a point on each lateral crus that is the new dome location. Hydrodissect the skin under this point 3 to $5 \mathrm{~mm}$ laterally and medially and carefully separate it from the undersurface of 
the crus. Next, place a suture through the cartilage to reestablish the new dome.

Aging changes of the lateral crus decrease its structural integrity and often result in external nasal valve collapse. Lateral crural strut grafts are an effective technique for supporting the lateral nasal wall. They are strips of cartilage placed between the lateral crus and the vestibular skin. Originally, they were described as an extension of the lateral crus crossing the piriform aperture to improve support of the external valve. ${ }^{33}$ More recently, these have been modified in both position and size. Many surgeons make them shorter and place them somewhat obliquely to the long axis of the lateral crus. ${ }^{34}$ Besides the structural improvement to the lateral crus, lateral crural strut grafts are an effective way to reshape the tip contour-decreasing the bulbosity often found in the aged nasal tip (-Fig. 6 ).

They are typically 20 to $30 \mathrm{~mm}$ long, $5 \mathrm{~mm}$ wide, and $1 \mathrm{~mm}$ thick. Beveling the edges that contact the vestibular skin will make them harder for the patient to palpate after surgery. Before placement, I mark the point of maximal concavity in the supra-alar crease. This is the target for the graft pocket. Lateral crural strut grafts can be placed through a cephalic or a caudal approach, but the cephalic approach is usually easier. First, make a conservative cephalic trim of the lateral crus and develop a subperichondrial pocket between the cartilage and the vestibular skin. The pocket should extend to the marking made earlier in the supra-alar crease. Make certain that there are no residual septations in the pocket that will prevent easy graft placement. Once the graft is inserted, it can be affixed to the lateral crus with one or two absorbable sutures. These sutures will also flatten the lateral crus. Finally, it is important to suture the opening of the pocket at the cephalic trim to restore a favorable orientation of the short axis of the lateral crus. ${ }^{35}$ Lateral crural strut grafts can also be placed through an endonasal approach. The technique is similar but the dissection under the cartilage begins along the caudal border by way of a marginal incision.

Because the suspensory ligaments in the nose deteriorate with age and are frequently transected during surgery, it is important to reconstitute or reinforce them with sutures during surgery. Probably the most important of these is to recreate the interdomal ligament by placing a suture between the cephalic edges of the domes. This will narrow the tip, ensure symmetry, and increase the stability of the tip tripod.

\section{Augmentation of the Radix}

As aging bone is resorbed the radix deepens. ${ }^{6}$ This can contribute to the appearance of a pseudo hump on the dorsum. Augmenting the radix will restore a more youthful relationship between the glabella and dorsum. Radix grafts can be made of cartilage or soft tissue. Perichondrium is an excellent material for grafting. Regardless of the graft choice, they are placed in a precise pocket under the skin. This is usually done in conjunction with a rhinoplasty but can also be preformed through an external approach using a small horizontal incision in a skin crease. ${ }^{36}$ When augmenting the radix as part of a rhinoplasty the graft can be placed loosely in the pocket, but I prefer to use a suture to guide it into the radix, passing the suture through the
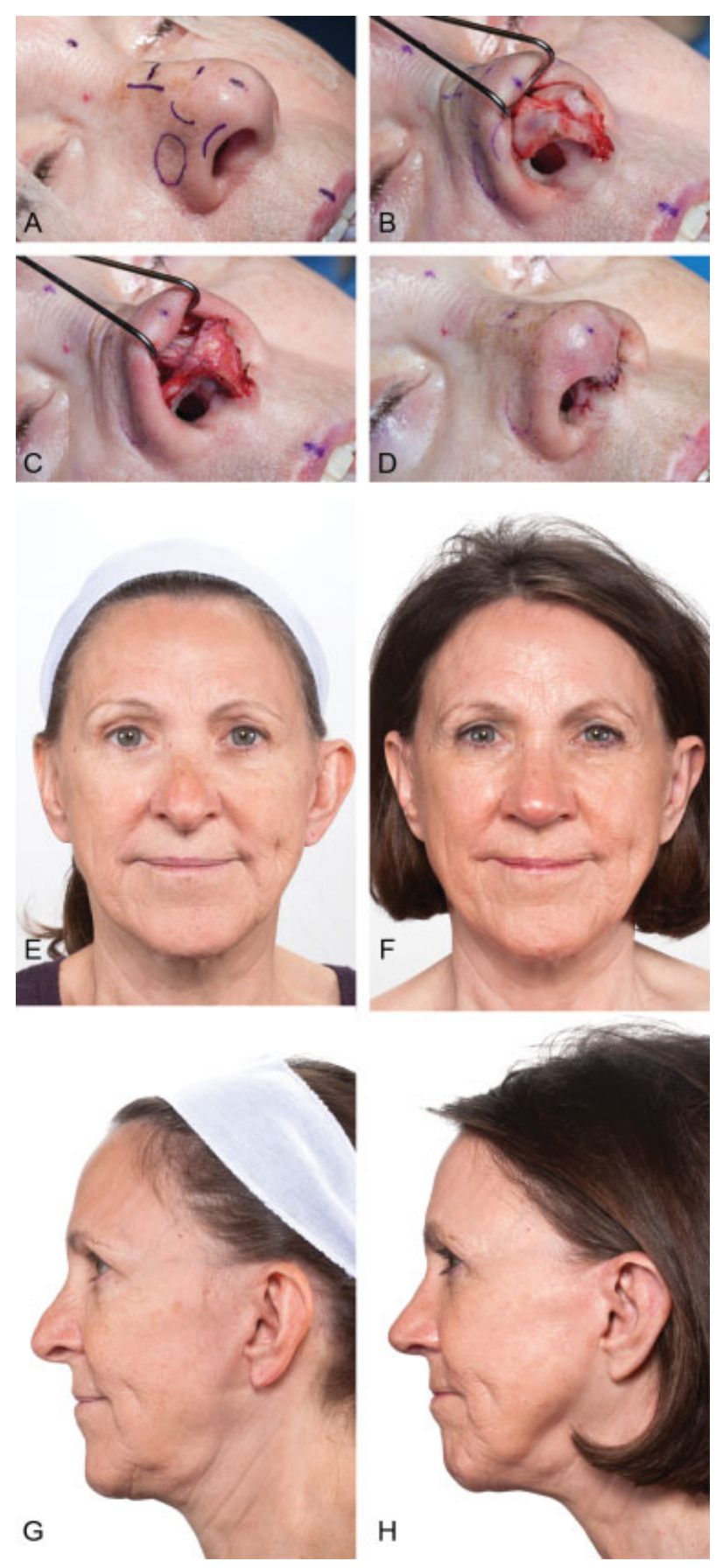

Fig. 6 (A) The figure shows an intraoperative surgeon's view of a patient with a dependent, round tip. (B, C) The figures show the lower lateral cartilages before and after placement of lateral crural strut grafts to reshape and reorient them. (D) This shows the immediate postoperative change. Note the improved tip definition and support of the alar margin. (E-H) The figures show the comparison between pre- and postoperative changes in this patient. The only surgery she has had on her face is the rhinoplasty.

skin to ensure accurate placement. The suture is taped to the skin and trimmed when the cast is removed.

\section{Augmentation of the Premaxilla}

Premaxillary augmentation is another technique to restore a more youthful skeletal foundation. Premaxillary grafts can be made of alloplastic or autologous materials and may be 
placed through vestibular or sublabial incisions. ${ }^{37}$ Through either of these approaches it is possible to dissect subperiosteal pockets laterally along the inferior border of the piriform aperture to accept placement of the graft. Leaving a small strip of periosteum in the midline near the anterior nasal spine provides a way to suture the implant or graft to the bone. The shape of the graft is critical to a good result. ${ }^{37}$ It must conform to the contours of the bone and be of appropriate size. Because the contour of the piriform aperture is a complex shape, preformed alloplastic implants are an easier option than carving autologous grafts.

\section{Rhinolift}

In 1990, Slavit et al from Mayo Clinic described the rhinolift operation..$^{38}$ This is a modified rhinoplasty with skin excision on the dorsum near the radix. Intercartilaginous incisions are made to release the lateral crura and permit cephalic rotation of the tip. An ellipse of redundant skin is excised, and the closure of the defect rotates the tip. They reported increased rotation and improved nasal breathing in their series of 20 patients.

\section{Conclusion}

The aging nose presents unique pathology due to the involutional changes that affect the cartilage and supporting structures. The cartilage becomes fragmented and partially replaced with fibrotic tissue and the suspensory ligaments show increasing laxity. The underlying skeletal foundation of the nose partially resorbs creating a retrusive platform and inadequate nasal support. These processes combine to decrease the projection and rotation of the nose often resulting in an increasingly prominent dorsal hump and worsened nasal breathing. Structural rhinoplasty techniques can restore the more youthful orientation of the nasal framework and alloplastic implants are useful adjuncts for replacing lost bone. More recently, minimally invasive options have shown some efficacy in the treatment of the aging nose and may play a larger role in the future. The involutional changes that affect the mandible, neck, and periorbita also affect the nose. A comprehensive facial rejuvenation plan should address these progressive effects.

\section{Conflict of Interest}

None declared.

\section{References}

1 Daniel RK, Palhazi P. The nasal ligaments and tip support in rhinoplasty: an anatomical study. Aesthet Surg J 2018;38(04): 357-368

2 Janeke JB, Wright WK. Studies on the support of the nasal tip. Arch Otolaryngol 1971;93(05):458-464

3 Gunter JP. Anatomical observations of the lower lateral cartilages. Arch Otolaryngol 1969;89(04):599-601

4 Pessa JE, Zadoo VP, Mutimer KL, et al. Relative maxillary retrusion as a natural consequence of aging: combining skeletal and softtissue changes into an integrated model of midfacial aging. Plast Reconstr Surg 1998;102(01):205-212
5 Mendelson BC, Hartley W, Scott M, McNab A, Granzow JW. Agerelated changes of the orbit and midcheek and the implications for facial rejuvenation. Aesthetic Plast Surg 2007;31(05):419-423

6 Shaw RB Jr, Kahn DM. Aging of the midface bony elements: a three-dimensional computed tomographic study. Plast Reconstr Surg 2007;119(02):675-681, discussion 682-683

7 Bartlett SP, Grossman R, Whitaker LA. Age-related changes of the craniofacial skeleton: an anthropometric and histologic analysis. Plast Reconstr Surg 1992;90(04):592-600

8 Garn SM, Rohmann CG, Wagner B, Ascoli W. Continuing bone growth throughout life: a general phenomenon. Am J Phys Anthropol 1967;26(03):313-317

9 Bolin A. Proximal alveolar bone loss in a longitudinal radiographic investigation. Swed Dent J Suppl 1986;35:1-108

10 Pessa JE, Peterson ML, Thompson JW, Cohran CS, Garza JR. Pyriform augmentation as an ancillary procedure in facial rejuvenation surgery. Plast Reconstr Surg 1999;103(02):683-686

11 Mendelson $\mathrm{B}$, Wong $\mathrm{CH}$. Changes in the facial skeleton with aging: implications and clinical applications in facial rejuvenation. Aesthetic Plast Surg 2012;36(04):753-760

12 Rohrich RJ, Hollier LH Jr, Janis JE, Kim J. Rhinoplasty with advancing age. Plast Reconstr Surg 2004;114(07):1936-1944

13 Edelstein DR. Aging of the normal nose in adults. Laryngoscope 1996;106(9 Pt 2):1-25

14 Helal HA, Ghanem MAM, Al-Badawy AM, Abdel Haleem MM, Mousa $\mathrm{MH}$. Histological and anthropometric changes in the aging nose. Aesthet Surg J 2019;39(09):943-952

15 Krmpotić-Nemanić J, Kostović I, Rudan P, Nemanić G Morphological and histological changes responsible for the droop of the nasal tip in advanced age. Acta Otolaryngol 1971;71(02):278-281

16 Gubisch W, Reichert H. Problems of rhinoplasty in the elderly. Handchir Mikrochir Plast Chir 1986;18(05):255-256

17 Khavkin J, Ellis DA. Aging skin: histology, physiology, and pathology. Facial Plast Surg Clin North Am 2011;19(02):229-234

18 Toriumi DM, Rosenberger E. Rhinoplasty of the aging nose. Facial Plast Surg 2016;32(01):59-69

19 Dick MK, Patel BC. Rhinophyma. Treasure Island, FL: StatPearls; 2020

20 Shulman O, Westreich M, Shulman J. Motivation for rhinoplasty: changes in 5970 cases, in three groups, 1964 to 1997. Aesthetic Plast Surg 1998;22(06):420-424

21 Bridger GP. Physiology of the nasal valve. Arch Otolaryngol 1970; 92(06):543-553

22 Cochran CS, Ducic Y, DeFatta RJ. Restorative rhinoplasty in the aging patient. Laryngoscope 2007;117(05):803-807

23 Eroğlu S, Cingi C, Hamilton GS. Suture lift of the nasal tip. In: Cingi C, Bayar Muluk N, Scadding GK, Mladina R, eds. Challenges in Rhinology. Cham: Springer; 2021:277-284

24 Saban Y, Javier B, Massa M. Nasal lift-nasal valve lift and nasal tip lift-preliminary results of a new technique using noninvasive selfretaining unidirectional nasal suspension with threads. Facial Plast Surg 2014;30(06):661-669

25 Tiryaki KT. Shuttle lifting of the nose: a minimally invasive approach for nose reshaping. Aesthet Surg J 2010;30(02):176-183

26 Kang SH, Moon SH, Kim HS. Nonsurgical rhinoplasty with polydioxanone threads and fillers. Dermatol Surg 2020;46(05): 664-670

27 Rezaee Khiabanloo S, Nabie R, Aalipour E. Outcomes in thread lift for face, neck, and nose: a prospective chart review study with APTOS. J Cosmet Dermatol 2020;19(11):2867-2876

28 Moon HJ. Injection rhinoplasty using filler. Facial Plast Surg Clin North Am 2018;26(03):323-330

29 Raspaldo H. Eiffel tower nose-lift: anatomical basis and concepts for safe and effective nasal injections. Plast Reconstr Surg Glob Open 2016;4(12):e1167

30 Wong BJF, Friedman O, Hamilton GS III. Grafting techniques in primary and revision rhinoplasty. Facial Plast Surg Clin North Am 2018;26(02):205-223 
31 Foda HM, Kridel RW. Lateral crural steal and lateral crural overlay: an objective evaluation. Arch Otolaryngol Head Neck Surg 1999; 125(12):1365-1370

32 Insalaco L, Rashes ER, Rubin SJ, Spiegel JH. Association of lateral crural overlay technique with strength of the lower lateral cartilages. JAMA Facial Plast Surg 2017;19(06):510-515

33 Gunter JP, Friedman RM. Lateral crural strut graft: technique and clinical applications in rhinoplasty. Plast Reconstr Surg 1997;99 (04):943-952, discussion 953-955

34 Cochran CS, Sieber DA. Extended alar contour grafts: an evolution of the lateral crural strut graft technique in rhinoplasty. Plast Reconstr Surg 2017;140(04):559e-567e
35 Hamilton GS III. Form and function of the nasal tip: reorienting and reshaping the lateral crus. Facial Plast Surg 2016;32(01): 49-58

36 Hamilton GS III. Dorsal failures: from saddle deformity to pollybeak. Facial Plast Surg 2018;34(03):261-269

37 Fanous N, Yoskovitch A. Premaxillary augmentation for central maxillary recession: an adjunct to rhinoplasty. Facial Plast Surg Clin North Am 2002;10(04):415-422

38 Slavit DH, Lipton RJ, Kern EB, McCaffrey TV. Rhinolift operation in the treatment of the aging nose. Otolaryngol Head Neck Surg 1990;103(03):462-467 